\title{
Biomechanical parameters of the cornea measured with the Ocular Response Analyzer in normal eyes
}

\author{
Aachal Kotecha ${ }^{1 *}$, Richard A Russell ${ }^{1}$, Angelos Sinapis ${ }^{1}$, Sayeh Pourjavan², Dimitros Sinapis ${ }^{1}$ \\ and David F Garway-Heath ${ }^{1}$
}

\begin{abstract}
Background: To evaluate the relationships between Reichert Ocular Response Analyzer (ORA) parameters corneal hysteresis $(\mathrm{CH})$ and corneal response factor (CRF) and ocular dimensions, age and intraocular pressure.

Methods: Two hundred and twelve eyes of 212 participants with no ocular pathology had CH and CRF measured with the ORA. Intraocular pressure (IOP) was measured with the Dynamic Contour tonometer and central corneal thickness (CCT) was also evaluated. Partial least squares linear regression (PLSLR) analyses were performed to examine the relationships between each response variable, $\mathrm{CH}$ and $\mathrm{CRF}$, and the predictor variables age, corneal curvature (CC), axial length (AL), CCT and IOP.

Results: $\mathrm{CH}$ was positively associated with CCT and negatively associated with age (scaled coefficients: CCT 0.62, $p<0.0001$; age $-0.55, p<0.0001 ; r^{2}=0.25$ ). CRF was positively associated with CCT and DCT IOP and negatively associated with age and AL (scaled coefficients: CCT 0.89, $p<0.0001$; DCT IOP 0.46, $p<0.01$; age $-0.60, p<0.0001$; $\mathrm{AL}-0.37, \mathrm{p}<0.01 ; \mathrm{r}^{2}=0.43$ ). There was no significant association between $\mathrm{CC}$ and $\mathrm{CH}$ or CRF.

Conclusions: The study suggests that age and CCT are strongly associated with $\mathrm{CH}$ and CRF, and that the latter is also influenced by AL and IOP. However, the variables studied could explain only $25 \%$ and $43 \%$ of the measured variation in $\mathrm{CH}$ and $\mathrm{CRF}$, respectively, suggesting other factors also affect the values of these measurements.
\end{abstract}

\section{Background}

Knowledge of the cornea's biomechanical properties is important for a wide variety of applications within ophthalmology. Corneal biomechanical variations are known to affect the accuracy of intraocular pressure measurements $[1,2]$, may be used to identify early corneal disease $[3,4]$, and may assist with predicting refractive outcomes following corneal refractive surgery [5]. It has also been suggested that corneal biomechanical properties may reflect globe biomechanics and thus give an indication of the susceptibility of developing glaucomatous damage $[6,7]$.

Until recently, most investigations evaluating corneal biomechanics were based on ex vivo tissue [8,9]. However,

\footnotetext{
*Correspondence: aachal.kotecha@ucl.ac.uk

${ }^{1} \mathrm{NIHR}$ Biomedical Research Centre for Ophthalmology, Moorfields Eye Hospital NHS Foundation Trust and UCL Institute of Ophthalmology, 11-43, Bath Street, London EC1V 9EL, UK

Full list of author information is available at the end of the article
}

the Reichert Ocular Response Analyzer (ORA; Reichert Ophthalmic Instruments, Buffalo, NY, USA) has facilitated an in vivo measurement of aspects of corneal biomechanical properties. The device measures the central corneal response to indentation by a rapid jet of air and provides two metrics of corneal biomechanics, corneal hysteresis $(\mathrm{CH})$ and the corneal response factor $(\mathrm{CRF})$. It is thought that $\mathrm{CH}$ predominantly reflects the viscous dampening properties of the cornea whilst CRF, a metric empirically derived to be strongly correlated with central corneal thickness (CCT), is thought to be most associated with the cornea's elastic response [10]. However, how these metrics relate to conventional biomechanical measurements is still relatively unclear. Studies examining the ORA biomechanical metrics have suggested associations with age, CCT and IOP [11,12]; however, some suggest no association with these parameters.

The purpose of this study was to evaluate the relationships between ORA generated corneal biomechanical 
characteristics and ocular dimensions, age and IOP in a cohort of normal eyes. Understanding the relationships between ORA measures and ocular dimensions may help better elucidate the significance of, and applications for, the metrics it produces.

\section{Methods}

This was a prospective cross-sectional observational study. The study had the approval of the local research ethical committee (REC; London-City Road and Hampstead REC and Riverside REC, London, United Kingdom) and informed consent, according to the tenets of the Declaration of Helsinki, was obtained from each participant prior to examination. Study participants were recruited from staff, spouses and friends of patients attending Moorfields Eye Hospital, London, UK, or St. Luc University Hospital, Université Catholique de Louvain, Belgium. Data was collected between October 2008 and April 2011. Participants underwent a complete ophthalmic investigation, including visual field testing with the Humphrey Visual Field Analyser (HFA: Zeiss Humphrey Systems, Dublin, California, USA) SITA standard threshold 24-2 strategy. Participants were excluded from the study if they had any signs of corneal pathology, corneal astigmatism $\geq 2$ dioptres or a history of incisional or intraocular surgery, a visual field defect, suspicious optic disc appearance, IOP $\geq 30 \mathrm{mmHg}$, a history of diabetes or a family history of glaucoma in a first degree relative. Soft-contact lens wearers were required to remove their lenses at least 24 hours prior to study participation; rigid contact lens wearers were excluded from the study. Of eligible participants, only one randomly chosen eye was measured for the study.

Study participants first had axial length (AL) and corneal curvature (CC) measurements made with the IOLMaster (version 3.01, Carl Zeiss Meditech AG, Jena, Germany). Prior to instillation of topical anaesthesia, participants underwent ORA measurements and three good quality waveform scans, defined as having symmetry in height between the two peaks of the waveform, were recorded and the mean value used in subsequent analysis.

Following instillation of topical corneal anaesthesia (proxymetacaine hydrochloride $0.5 \%$ with fluorescein sodium $0.25 \%$ ), IOP measurements were made using both the Goldmann applanation tonometer (GAT) and the dynamic contour tonometer (DCT). Two GAT IOP and three DCT IOP measurements were made in a randomised order. Only DCT measurements with a 'quality' reading of 1,2 or 3 were accepted and the first DCT reading was discarded in accordance with manufacturer's guidance. A minimum two-minute interval was left between IOP measurements with each device to minimise the tonographic effects of repeated tonometry measurements [13]. The mean of two IOP readings, taken with each of the two instruments, was calculated for each participant and was used in the analyses.
Measurements of CCT, using an ultrasound pachymeter (Altair, Optikron 2000, Roma, Italy), were made at the end of the visit; the average of 3 readings was recorded. All measurements were performed by one of four investigators (AK, AS, DS or SP).

Based on previous pilot data from an unrelated dataset, it was calculated that a sample of 194 eyes was required to achieve a correlation between $\mathrm{CH}$ and age of rho $=-0.2$, with $80 \%$ power at the $\mathrm{p}<0.05$ level.

\section{Data analysis}

Multivariate statistical analyses are effective tools to identify and explore the relationships between a response variable and several predictor variables. In this study, the response variable of interest was either $\mathrm{CH}$ or $\mathrm{CRF}$ and the predictor variables of interest were age, CC, CCT, IOP and AL. The theoretical assumptions of standard multiple linear regression (MLR) analysis limit its application to explore the relationships between $\mathrm{CH}, \mathrm{CRF}$ and the predictors for this study as the predictor variables are correlated, a statistical phenomenon known as multicollinearity. In circumstances where there is multicollinearity in the predictors, partial least squares linear regression (PLSLR) offers a robust alternative to MLR [14]. Like MLR, the objective of PLSLR is to describe the relationships between the response and predictor variables.

For this study, two PLSLR models were fitted to a subset of the data under investigation. The 'calibration' dataset consisted of a proportion (80\%) of participants randomly selected from the complete sample used to construct a predictive model for $\mathrm{CH}$ and CRF. The number of components in each PLSLR model was chosen using the 'leaveone-out method' [15]. The predictive performance of each model was then tested on the data excluded from the calibration data, which consisted of the remainder of participants. As the predictor variables have differing units of measurement, PLSLR models were also fitted using scaled predictor variables (where each predictor was weighted by dividing it by its standard deviation) in order to evaluate the relative impact of each variable on the response.

\section{Results}

Data were collected from 212 participants and the demographics of the cohort are presented in Table 1. The majority of participants were of Caucasian ethnicity $(n=167)$, followed by Indian $(n=29)$, Far-East Asian $(n=9)$ and African/Afro-Caribbean $(n=7)$.

\section{Correlations between $\mathrm{CH}, \mathrm{CRF}$ and other parameters}

As some of the data were non-normally distributed, Spearman's rank correlation test was used to evaluate correlations between parameters (Table 2). ORA corneal biomechanical parameters were positively correlated with ORA IOPcC and GAT IOP, and to a lesser extent 
Table 1 Demographics of study cohort

\begin{tabular}{lccc}
\hline Total cohort $\mathbf{n}=\mathbf{2 1 2}$ & Mean & SD & Range \\
\hline Eye (left/number) & 96 & & \\
Sex (male/number) & 95 & & \\
Age (years) & 50.4 & 19.0 & 19.0 to 92.6 \\
AL (mm) & 23.8 & 1.1 & 21.4 to 28.7 \\
CC (mm) & 7.7 & 0.3 & 7.1 to 8.6 \\
Corneal astigmatism (dioptres) & 0.8 & 0.5 & 0.0 to 4.0 \\
CCT (microns) & 550 & 31 & 490 to 633 \\
GAT (mmHg) & 14.8 & 3.3 & 6.0 to 25.5 \\
DCT (mmHg) & 16.2 & 2.6 & 9.7 to 25.0 \\
ORA IOPcc (mmHg) & 15.5 & 3.6 & 8.7 to 29.0
\end{tabular}

(Key: $A L=$ axial length, $C C=$ corneal curvature, $C C T=$ central corneal thickness, $G A T=$ Goldmann applanation tonometry, DCT = dynamic contour tonometry, $I O P C C=$ ORA corneal compensated IOP).

with DCT IOP. CH and CRF were negatively associated with age, axial length and corneal curvature, and positively correlated with CCT.

Previous work has shown the DCT to be less affected by variations in corneal biomechanical properties [16]; consequently, for this study, the DCT IOP measurement was used as a surrogate measure of 'true' IOP. Thus, in total, the impact of five predictor variables on $\mathrm{CH}$ and CRF were considered.

\section{PLSLR analysis: corneal hysteresis and corneal response factor models}

All statistical analyses were carried out in R, a freely available statistical package (http://www.r-project.org/). A random selection of 169 participants was used to calibrate the PLSLR model, and the results of cross-validation (using the leave-one-out method) are illustrated in Figure 1; the root mean squared error of prediction (RMSEP) is plotted against the number of components included in the fitted model. Four components minimise the RMSEP in the $\mathrm{CH}$ model (RMSEP = 1.46; Figure 1A), and four components minimise the RMSEP in the CRF model (RMSEP $=1.42$; Figure 1B). Therefore, four components were employed in the $\mathrm{CH}$ and CRF PLSLR predictive models, respectively.

For the $\mathrm{CH}$ model, four components explained $25 \%$ of the variance in $\mathrm{CH}$. For the CRF model, four components explained $43 \%$ of the variance in CRF.

The coefficients for the $\mathrm{CH}$ and CRF PLSLR models are shown in Tables 3 and 4, respectively. The data illustrates that age and CCT exerts a strong influence on $\mathrm{CH}$ and CRF, with the later also being associated with axial length and IOP.

Finally, the accuracy of the models to predict $\mathrm{CH}$ and CRF was tested using the independent validation data made up of 43 normal participants. The predicted value for each response is plotted against the observed value in Figures 2. The $\mathrm{CH}$ prediction model produced an $\mathrm{r}^{2}$ value of 0.16 and a RMSEP equal to 1.44 (see Figure 2A),

Table 2 Correlation table showing Spearman's rho and significance values for parameters

\begin{tabular}{|c|c|c|c|c|c|c|c|c|}
\hline $\begin{array}{l}\text { Spearman's rho } \\
\text { coefficient (p) }\end{array}$ & $\begin{array}{l}\text { Age } \\
\text { (yrs) }\end{array}$ & $\begin{array}{c}\mathrm{AL} \\
(\mathrm{mm})\end{array}$ & $\begin{array}{c}\mathrm{CC} \\
(\mathrm{mm})\end{array}$ & $\begin{array}{c}\mathrm{CCT} \\
\text { (microns) }\end{array}$ & $\begin{array}{l}\text { GAT IOP } \\
(\mathrm{mmHg})\end{array}$ & $\begin{array}{l}\text { DCT IOP } \\
\text { (mmHg) }\end{array}$ & $\begin{array}{c}\text { IOPcc } \\
(\mathrm{mmHg})\end{array}$ & $\begin{array}{c}\mathrm{CH} \\
(\mathrm{mmHg})\end{array}$ \\
\hline \multirow[t]{2}{*}{$\overline{\mathrm{AL}(\mathrm{mm})}$} & $-0.19^{\dagger}$ & & & & & & & \\
\hline & $(0.007)$ & & & & & & & \\
\hline \multirow[t]{2}{*}{$\mathrm{CC}(\mathrm{mm})$} & -0.01 & $0.45^{\dagger}$ & & & & & & \\
\hline & $(0.87)$ & $(<0.001)$ & & & & & & \\
\hline \multirow[t]{2}{*}{ CCT (microns) } & 0.12 & -0.04 & -0.01 & & & & & \\
\hline & $(0.09)$ & $(0.54)$ & (0.98) & & & & & \\
\hline \multirow[t]{2}{*}{ GAT IOP (mmHg) } & -0.06 & -0.01 & -0.08 & $0.20^{\dagger}$ & & & & \\
\hline & $(0.41)$ & $(0.84)$ & $(0.27)$ & $(<0.01)$ & & & & \\
\hline \multirow[t]{2}{*}{$\mathrm{DCT}$ IOP (mmHg) } & 0.06 & 0.07 & -0.01 & $0.20^{+}$ & $0.78^{\dagger}$ & & & \\
\hline & $(0.39)$ & $(0.34)$ & $(0.93)$ & $(<0.01)$ & $(<0.001)$ & & & \\
\hline \multirow[t]{2}{*}{ IOPcc $(\mathrm{mmHg})$} & $0.15^{\dagger}$ & 0.07 & 0.10 & $0.15^{\dagger}$ & $0.56^{\dagger}$ & $0.57^{\dagger}$ & & \\
\hline & $(0.03)$ & $(0.28)$ & $(0.16)$ & $(0.02)$ & $(<0.001)$ & $(<0.001)$ & & \\
\hline \multirow[t]{2}{*}{$\mathrm{CH}(\mathrm{mmHg})$} & $-0.19^{\dagger}$ & $-0.16^{\dagger}$ & $-0.16^{\dagger}$ & $0.36^{+}$ & $0.24^{\dagger}$ & $0.20^{\dagger}$ & $-0.26^{\dagger}$ & \\
\hline & $(<0.01)$ & $(0.02)$ & $(0.02)$ & $(<0.001)$ & $(<0.001)$ & $(<0.01)$ & $(<0.001)$ & \\
\hline \multirow[t]{2}{*}{$\mathrm{CRF}(\mathrm{mmHg})$} & $-0.22^{\dagger}$ & $-0.23^{\dagger}$ & $-0.16^{\dagger}$ & $0.47^{\dagger}$ & $0.28^{+}$ & $0.24^{\dagger}$ & $-0.11^{\dagger}$ & $0.74^{\dagger}$ \\
\hline & $(0.001)$ & $(0.002)$ & $(<0.01)$ & $(<0.001)$ & $(<0.001)$ & $(<0.001)$ & $(0.01)$ & $(<0.001)$ \\
\hline
\end{tabular}

(Key: $A L=$ axial length, $C C=$ corneal curvature, $C C T=$ central corneal thickness, GAT IOP = Goldmann applanation tonometry intraocular pressure, $D C T$ IOP = dynamic contour tonometry intraocular pressure, IOPCC = Ocular response analyzer corneal compensated intraocular pressure, $\mathrm{CH}=\mathrm{corneal}$ hysteresis, $\mathrm{CRF}=\mathrm{corneal}$ response factor, ${ }^{+}=$significant at the $p<0.05$ level). 

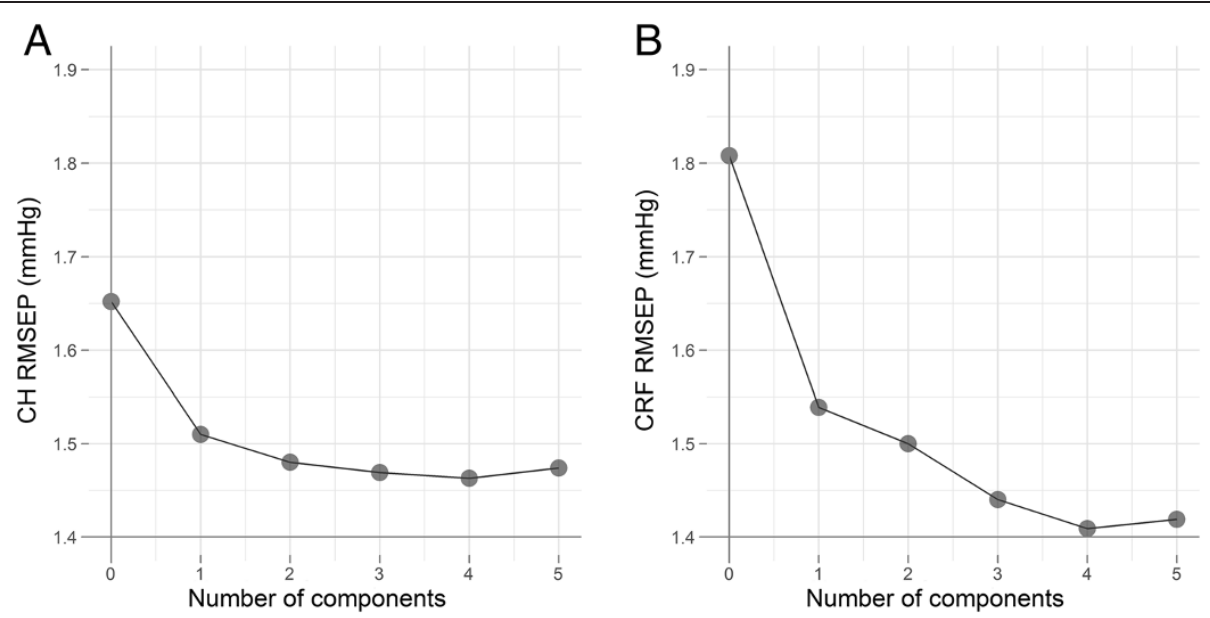

Figure 1 Cross-validated RMSEP curves for PLSLR models. These graphs illustrate the effect of the number of components on the precision of the $\mathrm{CH}(\mathbf{A})$ and $\mathrm{CRF}(\mathbf{B})$ regression models. In both models, the prediction error was minimized using four components.

while the CRF prediction model gave rise to an $r^{2}$ value of 0.38 and a RMSEP equal to 1.24 (see Figure 2B).

\section{Discussion}

The results of this study suggest that ORA-generated metrics of corneal biomechanical properties in nonglaucomatous eyes are correlated with age, CCT and, in the case of CRF, AL. The relationships between these variables are complex and the predictor variables do not explain all the variation in $\mathrm{CH}$ or CRF measurements.

The data were analysed with PLSLR analysis since the predictor variables were correlated. In PLSLR, components (known as 'latent variables') are extracted from the predictor variables by maximising the covariance between the predictor variables and the response variable.

It has been suggested that $\mathrm{CH}$ represents the viscoelastic capacity of the cornea, that is, the cornea's ability to dampen and dissipate applied energy. Hysteresis is dependent on the relative contributions of both elasticity and viscosity, and it has been shown that alterations in either component will have very different and sometimes

Table 3 Coefficients of PLSLR predictive model for $\mathrm{CH}$ in the calibration dataset

\begin{tabular}{lccc}
\hline $\begin{array}{l}\text { Predictor } \\
\text { variable }\end{array}$ & $\begin{array}{c}\text { PLSLR coefficient } \\
\text { (non-scaled) }\end{array}$ & $\begin{array}{c}\text { PLSLR coefficient } \\
\text { (scaled) }\end{array}$ & P value \\
\hline AL (mm) & -0.23 & -0.22 & 0.05 \\
CC (mm) & -0.02 & -0.02 & 0.85 \\
CCT (microns) & 0.02 & 0.62 & $<0.0001$ \\
Age (years) & -0.03 & -0.55 & $<0.0001$ \\
DCT IOP & 0.09 & 0.24 & 0.05 \\
(mmHg) & & & \\
\hline
\end{tabular}

The scaled coefficients illustrate that CCT and age have the most significant impact on $C H$. (Key: $A L=$ axial length, $C C=$ corneal curvature, $C C T=$ central corneal thickness, $D C T I O P=$ dynamic contour tonometry intraocular pressure). opposing effects on measured hysteresis [17]. Our finding that $\mathrm{CH}$ reduces with age corroborates the findings of experimental ex vivo studies that show an increase in collagen cross-linking with age [18], which result in a reduction in the viscosity of the cornea and thus an increase in the 'stiffness' of the structure $[19,20]$. The data are also in agreement with previous clinical work evaluating the effect of age on ORA measured corneal biomechanical properties $[11,12,21]$. Taken together, these findings suggest that aging results in an overall reduction in the dampening capacity of the cornea.

Our study also agrees with previous findings that $\mathrm{CH}$ is positively associated with CCT $[11,21]$. This is unsurprising as one might expect a thicker cornea to have a greater viscous dampening capacity. Recent work has also found that $\mathrm{CH}$ reduces with increasing axial length [22], and it has been suggested that these findings are indicative of altered biomechanics in axially myopic eyes. However, other reports have found no association between axial length and $\mathrm{CH}$ [23]. In the present study, Spearman's correlation

Table 4 Coefficients of PLSLR predictive model for CRF in the calibration dataset

\begin{tabular}{lccc}
\hline $\begin{array}{l}\text { Predictor } \\
\text { variable }\end{array}$ & $\begin{array}{c}\text { PLSLR coefficient } \\
\text { (non-scaled) }\end{array}$ & $\begin{array}{c}\text { Scaled PLSLR } \\
\text { coefficient }\end{array}$ & P value \\
\hline AL $(\mathrm{mm})$ & -0.39 & -0.37 & $<0.01$ \\
CC (mm) & -0.03 & -0.04 & 0.74 \\
CCT (microns) & 0.03 & 0.89 & $<0.0001$ \\
Age (years) & -0.03 & -0.60 & $<0.0001$ \\
DCT IOP & 0.18 & 0.46 & $<0.01$ \\
(mmHg) & & &
\end{tabular}

The scaled coefficients illustrate that CCT has the greatest impact on CRF, followed by age, IOP and $A L$. (Key: $A L=$ axial length, $C C=$ corneal curvature, $C C T=$ central corneal thickness, DCT IOP = dynamic contour tonometry intraocular pressure). 

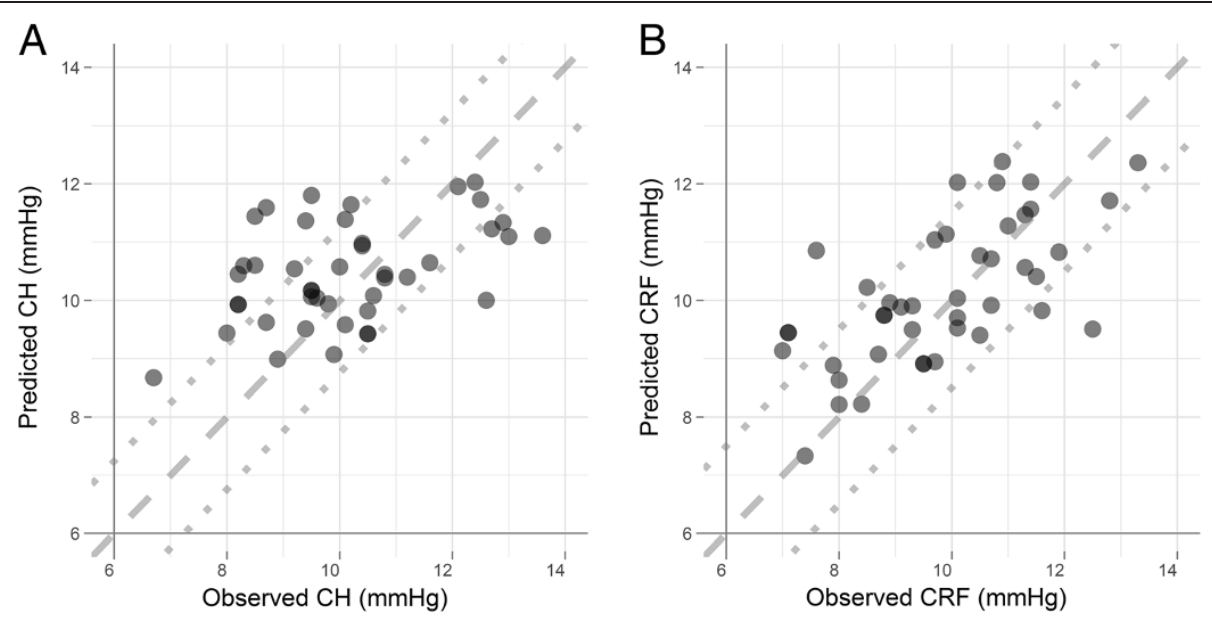

Figure 2 Scatterplot showing the prediction performance of the PLSLR in the test dataset. Graphs show the PLSLR prediction performance of $\mathrm{CH}(\mathbf{A})$ and $\mathrm{CRF}(\mathbf{B})$. The dashed line represents the line of unity. If the prediction model was $100 \%$ accurate, all data points would fall on this line. The dotted lines indicate the 95\% confidence limits of the Normal distribution; these are \pm 1.24 and \pm 1.50 for $\mathrm{CH}$ and CRF, respectively. Compared with the $\mathrm{CH}$ model, predicted values of CRF are close to observed values, indicating that the studied variables can explain a large proportion of the variation in CRF measurements.

test suggested that $\mathrm{CH}$ was negatively associated with both axial length and corneal curvature; however, in the PLSLR model, these parameters were not significant (although axial length approached significance). These findings may be a reflection of the ethnic differences in the cohorts studied; our participants were predominantly of Caucasian ethnicity, whilst those in the other studies were of Far East Asian origin. Surprisingly, Spearman's correlation test also suggested that $\mathrm{CH}$ was positively associated with DCT IOP and GAT IOP; however, in the PLSLR model the association between DCT IOP and CH only approached significance. This suggests that the univariate association was either a spurious finding, or as a result of correlations with other predictor variables that were subsequently dealt with in the PLSLR model. Further work is required to establish the significance of relationships that may exist between axial length, IOP and $\mathrm{CH}$.

CRF was also found to increase with CCT and reduce with age. CRF was intended to quantify the overall corneal viscoelastic resistance to indentation and was developed to be strongly associated with CCT [24]. The fact that CRF reduces with age is counterintuitive, as it might be expected that the increase in corneal 'stiffness' resultant from an age-related increase in corneal collagen cross-linking would result in an increased resistance to deformation; however, our results do agree with previous findings [25]. The CRF represents a metric of corneal resistance to a near instantaneous indentation force applied axially. Our data suggest that the assumption that CRF reflects overall corneal rigidity may be an oversimplification and that other factors need to be considered when interpreting its value.
In the PLSLR model, CRF was also found to be positively associated with IOP. The cornea is hyperelastic; thus, the 'stretching' of the cornea under high IOP conditions will render it a 'stiffer' structure. Of interest is the finding that a lower CRF was associated with longer axial length, which was highlighted in the PLSLR model. This agrees with some [26] but not others' [22] findings. It is not clear what this relationship between CRF and axial length biomechanically represents. One may postulate that if CRF represents overall corneal rigidity, since longer eyes may have altered scleral biomechanics [27] a reduction in CRF may be an expected finding, in that a reduced corneal rigidity would be associated with a reduced scleral rigidity. However, further work is required to establish the significance of the relationship and how corneal biomechanics relate, if at all, to scleral biomechanics.

Of note is the proportion of variance in corneal biomechanical measurements predicted by the studied parameters when all the inter-relationships are considered. In the calibration PLSLR model, only $25 \%$ of the variation in measured $\mathrm{CH}$ could be explained by the variables studied, which reduced even further when applied to the test dataset. Whilst part of this may indicate true inter-individual variations in $\mathrm{CH}$ measurements, there may be other explanations for this observation. It is possible that this reflects a reduced signal-to-noise ratio in the $\mathrm{CH}$ measurement which masks the true effect of the predictors studied [28]. Furthermore, there may be significant and as yet unmeasured variables that effect the $\mathrm{CH}$ measurement. These may include the degree of corneal indentation and the rate and maximal level of external air pressure application, all of which have been 
shown experimentally to affect hysteresis $[17,29]$. In contrast, approximately $43 \%$ of CRF variation could be explained by the variables studied. This suggests that the CRF measurement can be characterised by CCT, age, IOP and AL. However, a large proportion of the measurement variation cannot be explained by these variables and may represent a combination of actual inter-individual CRF differences, measurement noise and other as yet undetermined factors.

\section{Conclusion}

In conclusion, this study of corneal biomechanics in normal eyes describes the complex interactions between ocular characteristics and ORA metrics, and finds that both age and CCT are significantly associated with $\mathrm{CH}$ and CRF. However, only a proportion of the variation in both these ORA metrics could be described by the ocular characteristics measured, implying that there are other elements contributing to the $\mathrm{CH}$ and $\mathrm{CRF}$ measurement.

Finally, condensing corneal biomechanical measures to a single summary metric will never completely describe the cornea's properties. Recently, investigators evaluating the ORA applanation signal have found that variations in specific signal elements are better descriptors of the corneal response to indentation particularly following refractive surgery procedures [30,31]. Further work is required to establish the validity of these new parameters and how they relate to more conventional biomechanical measures.

\section{Competing interests}

The authors declare that they have no competing interests.

\section{Authors' contributions}

AK: study design, data acquisition, statistical analysis, data interpretation, manuscript writing and final approval. RAR: statistical analysis, data interpretation, manuscript critique and final approval. AS: data acquisition, manuscript final approval. SP: data acquisition, manuscript critique and final approval. DS: data acquisition, manuscript final approval. DGH: study design, data interpretation, manuscript critique and final approval. All authors read and approved the final manuscript.

\section{Acknowledgements}

The authors would like to thank Reichert Corporation for the loan of the ORA. Drs Kotecha and Russell and Professor Garway-Heath have received a proportion of their funding from the Department of Health's National Institute for Health Research Biomedical Research Centre for Ophthalmology at Moorfields Eye Hospital NHS Foundation Trust and the UCL Institute of Ophthalmology. Presented in part at the Association for Research in Vision and Ophthalmology meeting, Fort Lauderdale, USA, May 2010.

\section{Author details}

${ }^{1} \mathrm{NIHR}$ Biomedical Research Centre for Ophthalmology, Moorfields Eye Hospital NHS Foundation Trust and UCL Institute of Ophthalmology, 11-43, Bath Street, London EC1V 9EL, UK. ²St. Luc University Hospital, Université Catholique de Louvain, Brussels, Belgium.

Received: 5 July 2013 Accepted: 24 January 2014

Published: 30 January 2014

\section{References}

1. Lau W, Pye D: Changes in corneal biomechanics and applanation tonometry with induced corneal swelling. Invest Ophthalmol Vis Sci 2011, 52(6):3207-3214.

2. Tonnu PA, Ho T, Newson T, El Sheikh A, Sharma K, White E, Bunce C, Garway-Heath D: The influence of central corneal thickness and age on intraocular pressure measured by pneumotonometry, non-contact tonometry, the Tono-Pen $\mathrm{XL}$, and Goldmann applanation tonometry. Br J Ophthalmol 2005, 89(7):851-854.

3. Schweitzer C, Roberts CJ, Mahmoud AM, Colin J, Maurice-Tison S, Kerautret J: Screening of forme fruste keratoconus with the ocular response analyzer. Invest Ophthalmol Vis Sci 2010, 51(5):2403-2410.

4. del Buey MA, Cristobal JA, Ascaso FJ, Lavilla L, Lanchares E: Biomechanical properties of the cornea in Fuchs' corneal dystrophy. Invest Ophthalmol Vis Sci 2009, 50(7):3199-3202.

5. Roberts C: Biomechanics of the cornea and wavefront-guided laser refractive surgery. J Refract Surg 2002, 18(5):S589-S592.

6. Congdon NG, Broman AT, Bandeen-Roche K, Grover D, Quigley HA: Central corneal thickness and corneal hysteresis associated with glaucoma damage. Am J Ophthalmol 2006, 141(5):868-875.

7. Wells AP, Garway-Heath DT, Poostchi A, Wong T, Chan KC, Sachdev N: Corneal biomechanics and optic nerve head compliance: corneal hysteresis but Not corneal thickness correlates with optic nerve surface deformation in glaucoma patients. Invest Ophthalmol Vis Sci 2008, 49(8):3262-3268

8. Dupps WJ Jr, Wilson SE: Biomechanics and wound healing in the cornea. Exp Eye Res 2006, 83(4):709-720.

9. Dupps WJ Jr: Hysteresis: new mechanospeak for the ophthalmologist. J Cataract Refract Surg 2007, 33(9):1499-1501.

10. Kotecha A: What biomechanical properties of the cornea are relevant for the clinician? Surv Ophthalmol 2007, 52(Suppl 2):S109-S114.

11. Kotecha A, Elsheikh A, Roberts CR, Zhu H, Garway-Heath DF: Corneal thickness- and age-related biomechanical properties of the cornea measured with the ocular response analyzer. Invest Ophthalmol Vis Sci 2006, 47(12):5337-5347.

12. Leite MT, Alencar LM, Gore C, Weinreb RN, Sample PA, Zangwill LM Medeiros FA: Comparison of corneal biomechanical properties between healthy blacks and whites using the Ocular Response Analyzer. Am J Ophthalmol 2010, 150(2):163-168. e161.

13. Recep OF, Hasiripi H, Vayisoglu E, Kalayci D, Sarikatipoglu H: Accurate time interval in repeated tonometry. Acta Ophthalmol Scand 1998, 76(5):603-605.

14. Wold H: Estimation of Principal Components and Related Models by Iterative Least Squares. In Multivariate Analysis. Edited by Krishnaiah PR. New York: Academic; 1966.

15. Lachenbruch PA, Mickey MR: Estimation of error rates in discriminant analysis. Technometrics 1968, 10(1):1-11.

16. Boehm AG, Weber A, Pillunat LE, Koch R, Spoerl E: Dynamic contour tonometry in comparison to intracameral IOP measurements. Invest Ophthalmol Vis Sci 2008, 49(6):2472-2477.

17. Glass DH, Roberts CJ, Litsky AS, Weber PA: A viscoelastic biomechanical model of the cornea describing the effect of viscosity and elasticity on hysteresis. Invest Ophthalmol Vis Sci 2008, 49(9):3919-3926.

18. Daxer A, Misof K, Grabner B, Ettl A, Fratzl P: Collagen fibrils in the human corneal stroma: structure and aging. Invest Ophthalmol Vis Sci 1998, 39(3):644-648

19. Elsheikh A, Wang D, Brown M, Rama P, Campanelli M, Pye D: Assessment of corneal biomechanical properties and their variation with age. Curr Eye Res 2007, 32(1):11-19.

20. Knox Cartwright NE, Tyrer JR, Marshall J: Age-related differences in the elasticity of the human cornea. Invest Ophthalmol Vis Sci 2011, 52(7):4324-4329.

21. Lau W, Pye D: A clinical description of Ocular Response Analyser measurements. Invest Ophthalmo/ Vis Sci 2011, 52(6):2911-2916.

22. Narayanaswamy A, Chung RS, Wu RY, Park J, Wong WL, Saw SM, Wong TY, Aung T: Determinants of corneal biomechanical properties in an adult chinese population. Ophthalmology 2011, 118(7):1253-1259.

23. Lim L, Gazzard G, Chan YH, Fong A, Kotecha A, Sim EL, Tan D, Tong L, Saw SM: Cornea biomechanical characteristics and their correlates with refractive error in Singaporean children. Invest Ophthalmol Vis Sci 2008 , 49(9):3852-3857. 
24. Methodology For Corneal Compensated lop And Corneal Resistance Factor For An Ocular Response Analyzer. Invest Ophthalmol Vis Sci 2006, 47:E-Abstract 2266.

25. Fontes BM, Ambrosio R Jr, Alonso RS, Jardim D, Velarde GC, Nose W: Corneal biomechanical metrics in eyes with refraction of -19.00 to +9.00 D in healthy Brazilian patients. J Refract Surg 2008, 24(9):941-945.

26. Chang PY, Chang SW, Wang JY: Assessment of corneal biomechanical properties and intraocular pressure with the Ocular Response Analyzer in childhood myopia. Br J Ophthalmol 2010, 94(7):877-881.

27. McBrien NA, Jobling Al, Gentle A: Biomechanics of the sclera in myopia: extracellular and cellular factors. Optom Vis Sci 2009, 86(1):E23-E30.

28. Kotecha A, Crabb DP, Spratt A, Garway-Heath DF: The relationship between diurnal variations in intraocular pressure measurements and central corneal thickness and corneal hysteresis. Invest Ophthalmol Vis Sci 2009, 50(9):4229-4236

29. Elsheikh A, Wang D, Rama P, Campanelli M, Garway-Heath D: Experimental assessment of human corneal hysteresis. Curr Eye Res 2008, 33(3):205-213.

30. Mikielewicz M, Kotliar K, Barraquer RI, Michael R: Air-pulse corneal applanation signal curve parameters for the characterisation of keratoconus. Br J Ophthalmol 2011, 95(6):793-798.

31. Kerautret J, Colin J, Touboul D, Roberts C: Biomechanical characteristics of the ectatic cornea. J Cataract Refract Surg 2008, 34(3):510-513.

\section{doi:10.1186/1471-2415-14-11}

Cite this article as: Kotecha et al.: Biomechanical parameters of the cornea measured with the Ocular Response Analyzer in normal eyes. BMC Ophthalmology 2014 14:11.

\section{Submit your next manuscript to BioMed Central and take full advantage of:}

- Convenient online submission

- Thorough peer review

- No space constraints or color figure charges

- Immediate publication on acceptance

- Inclusion in PubMed, CAS, Scopus and Google Scholar

- Research which is freely available for redistribution 